\section{Description and First Evaluation of Colonoscopy Technique with a New External Straightener of the Colon}

The most common difficulty encountered during colonoscopy is recurrent looping of the colonoscope in a long and mobile colon [1]. In an attempt to solve this problem most operators use a combination of straightening the endoscope and shortening the colon, via manual compression of the patient's abdomen by a second operator. This compression, however, cannot be constant and equal on different abdominal sites, and its efficacy can be impaired by the thickness of the abdominal and intestinal wall. To avoid these problems we have utilized a new device that we have named the External Straightener for Colonoscopy (ESC: Advantec, Milan, Italy).

The ESC is a hard ovoid-shaped $(30 \times 20 \mathrm{~cm})$, weighing $3.8 \mathrm{~kg}$, with a blunted beak-like protuberance at one pole (Figure 1). The main effect of this device is to enable a constant and efficient abdominal self-compression by the patient's own weight: the patient leans against the ESC in a left lateral semiprone recumbent position, with the left leg straight and the right one bent.

Colonoscopy using the ESC. The ESC is positioned in the left bottom quadrant of the abdomen, only when the colonoscope has reached the sigmoid-descending junction of the colon, with its longitudinal axis parallel to the axis of the sigmoid colon and the protuberance near the median line,

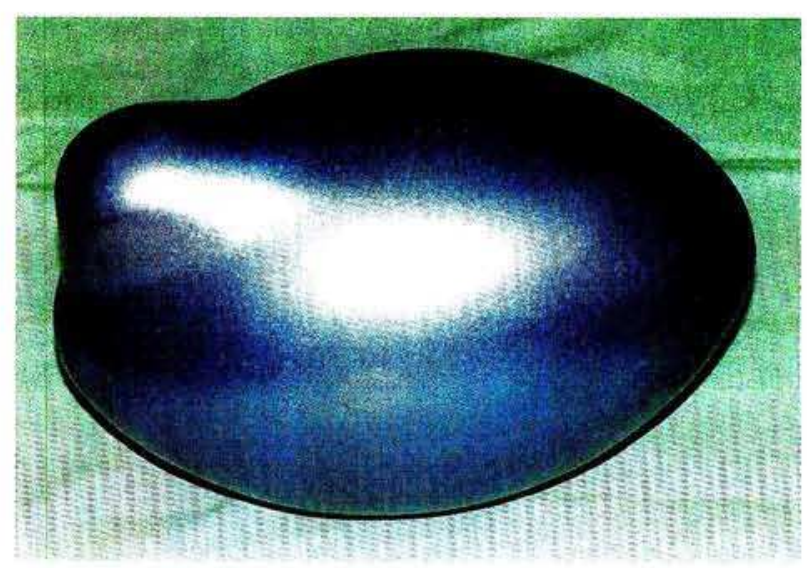

Figure 1 External straightener for colonoscopy (ESC)

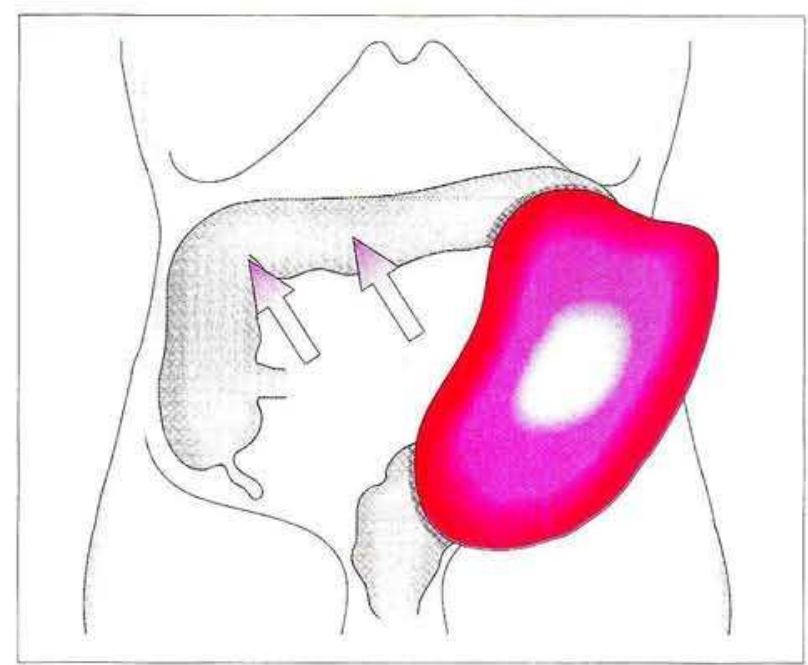

Figure 2 The ESC use in colonoscopy

under the navel. In order to straighten the sigmoid colon, lining it up with the descending colon, the colonoscope, with the up-down knob free and the right-left one locked, is simultaneously withdrawn and rotated counterclockwise (usually more than $\left(80^{\circ}\right.$ ). The pressure exerted by the ESC keeps the sigmoid colon stable in the 


\section{Unusual Cases and Technical Notes S107}

new position and allows progress upwards. When there is difficulty in progressing the scope, usually in the left angle, in a ptotic transverse colon, or in the right angle, the ESC position is modified. In this case the pole with the protuberance can be rotated on the abdominal wall to support the left angle, the transverse ptosis, or the right angle, with the compression on the sigmoid exerted by the other end of the device always remaining constant (Figure 2).

In our experience the use of the ESC produces several advantages: the cecum is reached in almost all cases, the examination time is shortened, and there is less discomfort and pain for the patient.
M. Paternuosto ', G. Cavallo²,

M. E. Bottiglieri', G. Migliore', G. Piai ${ }^{3}$

' Servizio Autonomo di Gastroenterologia ed Endoscopia Digestiva,

Presidio Ospedaliero Marcianise,

A.S.L. CE1, Marcianise, Italy

${ }^{2}$ Cattedra di Gastroenterologia,

Facoltà di Medicina,

Il Università di Napoli, Italy

${ }^{3}$ Divisione di Medicina,

Presidio Ospedaliero Marcianise,

A.S.L. CE1, Marcianise, Italy

\section{Reference}

1. Saunders BP, Macrae F, Williams CB What makes colonoscopy difficult? Gut 1993; 34: 1
Corresponding Author

M. Paternuosto, M.D.

Servizio Autonomo

di Gastroenterologia

ed Endoscopia Digestiva

Presidio Ospedaliero Marcianise, A.S.L.

CE

80125 Marcianise (CE)

Caserta

Italy

Fax: $\quad+39(823) 839579$

E-mail: mbottig1@tin.it 